\title{
Screen printing to create 3D tissue models
}

Narendra Pandala, Sydney Haywood, Michael A. LaScola, Adam Day, Joshua Leckron, Erin

Lavik* $^{*}$

Chemical, Biochemical and Environmental Engineering, University of Maryland, Baltimore

County, MD 21250, Piscataway Territories

\section{Supplementary Information}

\section{Screen Assembly}

The screen assembly begins with a wooden frame of size 4"x6" (Supplementary Figure -1A). The wooden frame is reinforced using $1 / 4$ " staples (Arrow T50, USA) to maintain the structural rigidity of the frame in the autoclaving process (Supplementary Figure -1B). A silkscreen of mesh size $125 \mu \mathrm{m}(43 \mathrm{~T}, 110 \mathrm{US})$ is then stretched on to the wooden frame and then attached to the frame using the 1/4" staples (Supplementary Figure -1C). The empty screen is then cleaned using $70 \%$ ethanol solution to degrease the mesh. The emulsion (Ulano-925WR-P) is then sensitized (according to the manufactures instructions) by dissolving the sensitizer in the water and mixing it in the emulsion thoroughly until an emulsion of uniform color is obtained. The emulsion is then let to set for an hour, the sensitized emulsion can be stored in dark place at room temperature for 2 months. The emulsion is then coated on to the mesh (Supplementary Figure -1D). The emulsion coated screen is dried overnight in a dark place. The photomasks are designed in Adobe illustrator with the accurate pixel density corresponding to the scale and the printer resolution. The photomasks are then printed on to a transparent OHP sheet using saturated black. The photomasks are then placed on dried emulsion coated screens at approximately $15 \mathrm{~cm}$ away from a UV lamp (Black-Ray, UVP B100, $365 \mathrm{~nm}$ ) for 3-5 minutes. The photomask is then removed, and the screens are washed using a stream of water until the non-hardened emulsion from the patterns is completely washed away, the screens are then set to dry (Supplementary Figure -1E). The screens are then placed in an autoclave bag and sterilized to be using in a tissue culture hood. Once the screens are used to print, the screens are washed with water, dried and autoclaved to be reused. 


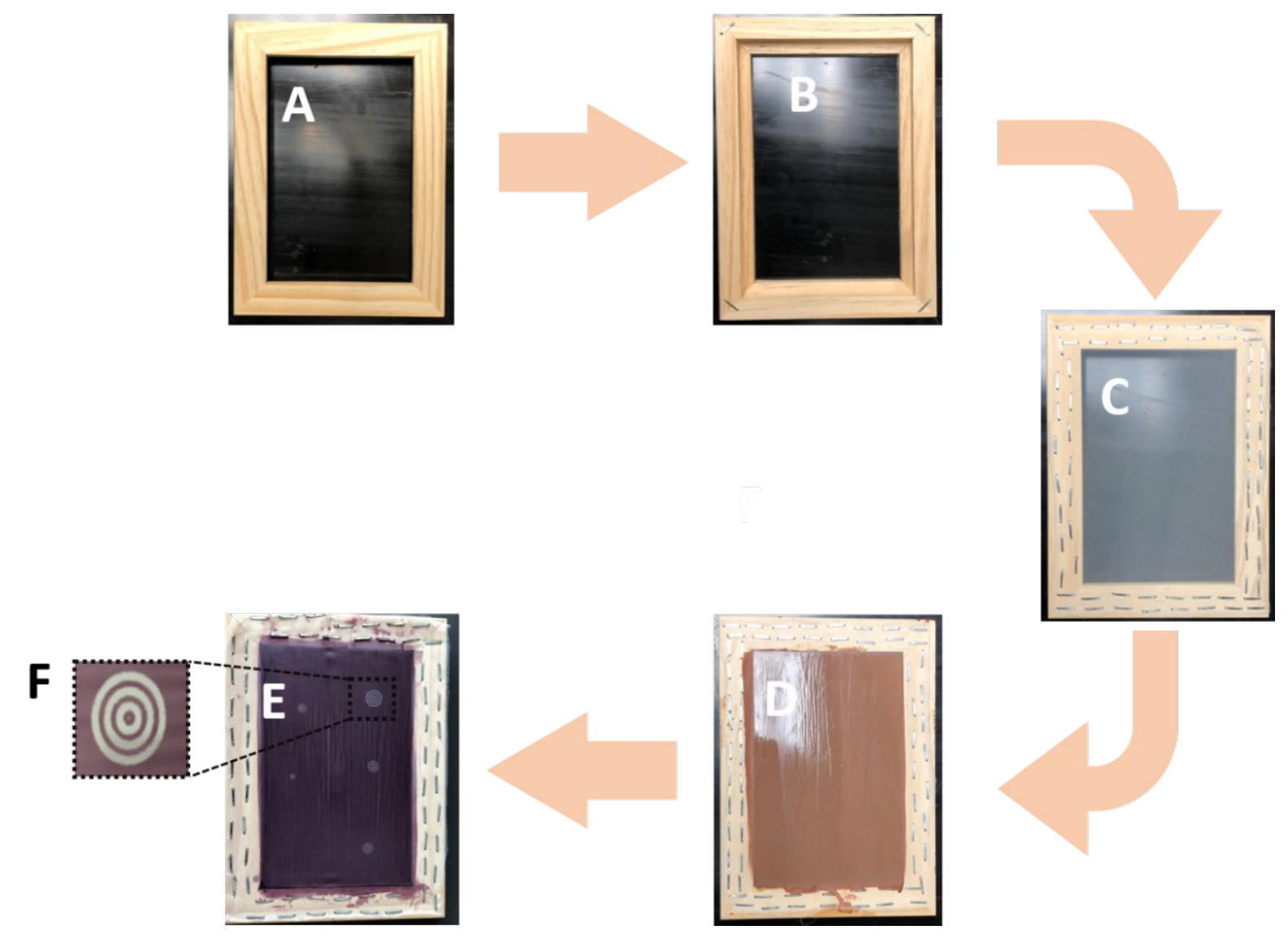

Supplementary Figure 1: A) Empty wooden screen B) wooden frame reinforced with staples C) Silkscreen stretched and attached to the wooden frame D) sensitized emulsion coated screen E) Assembled screen with a concentric circle pattern of various sizes.

\section{Sterilizing the screens:}

To sterilize the screens and to make them suitable for use in a tissue culture hood, we assembled the screens using wooden frames reinforced with $1 / 4$ " staples (Arrow T50, USA). The completely assembled and photomasked screens are autoclaved at a temperature of $121^{\circ} \mathrm{C}$ and a pressure of 31Psig for 15 minutes and 2 minutes of dry time (Gravity-1 setting) in an autoclave. Supplementary Figure 1 shows the images of the screens pre and post autoclave cycle, the screens remained structurally intact, and the pattern on the mesh and the mesh itself maintained its tension, making them suitable for printing after the autoclave cycle. This shows that after each print, the screens can be cleaned and then sterilized using an autoclave making them suitable for aseptic use. 

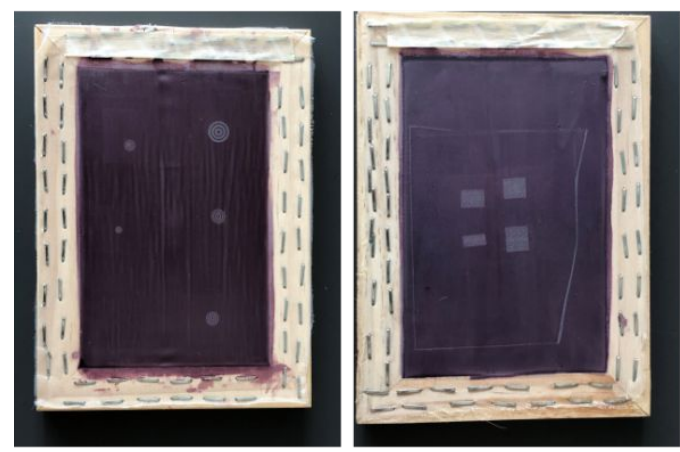

PREAUTOCLAVED SCREENS
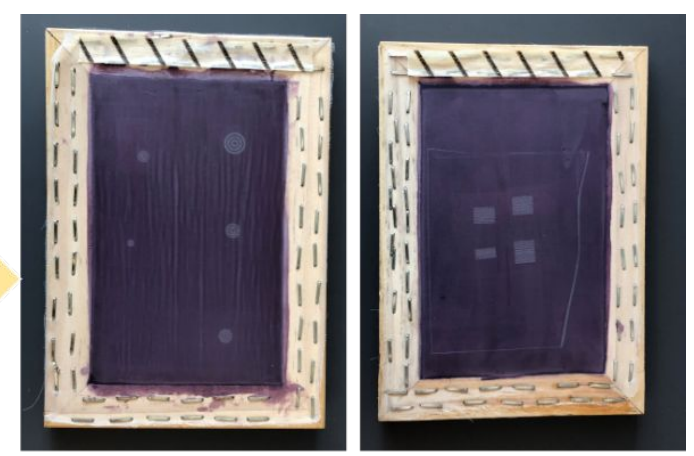

AUTOCLAVED SCREENS

Supplementary Figure 2: Pre and post autoclaved screens, showing autoclaving the screens doesn't alter their structural integrity, making them suitable for integration in an aseptic process

\section{Size of the prints on the transparent sheets:}

In the initial stages of the screen printing the patterns are designed on Adobe Illustrator and then printed on to inkjet printer compatible OHP sheets using an inkjet printer (Epson, WF-3640). The size of the patterns on the transparent sheets are measured using the laser confocal scanning microscope VK-X1000 (Keyence, Japan) and the results are analyzed using the Multifile analyzer software (Keyence, Japan). Supplementary Figure 2 shows the size of the patterns on the OHP sheets. From this it can be observed that the inkjet printer results in a size variation of about 70 $\mu \mathrm{m}$ in comparison to the designed pattern, which is carried on to the later stages of the printing process leading to a variation of approximately $90 \mu \mathrm{m}$ in the size of the prints. This can be adjusted by changing the size of the patterns in the Illustrator and then printing them to adjust for the variation in the sizes on the transparent sheets and there by adjusting the size of the patterns on the screens and finally the size of the prints.

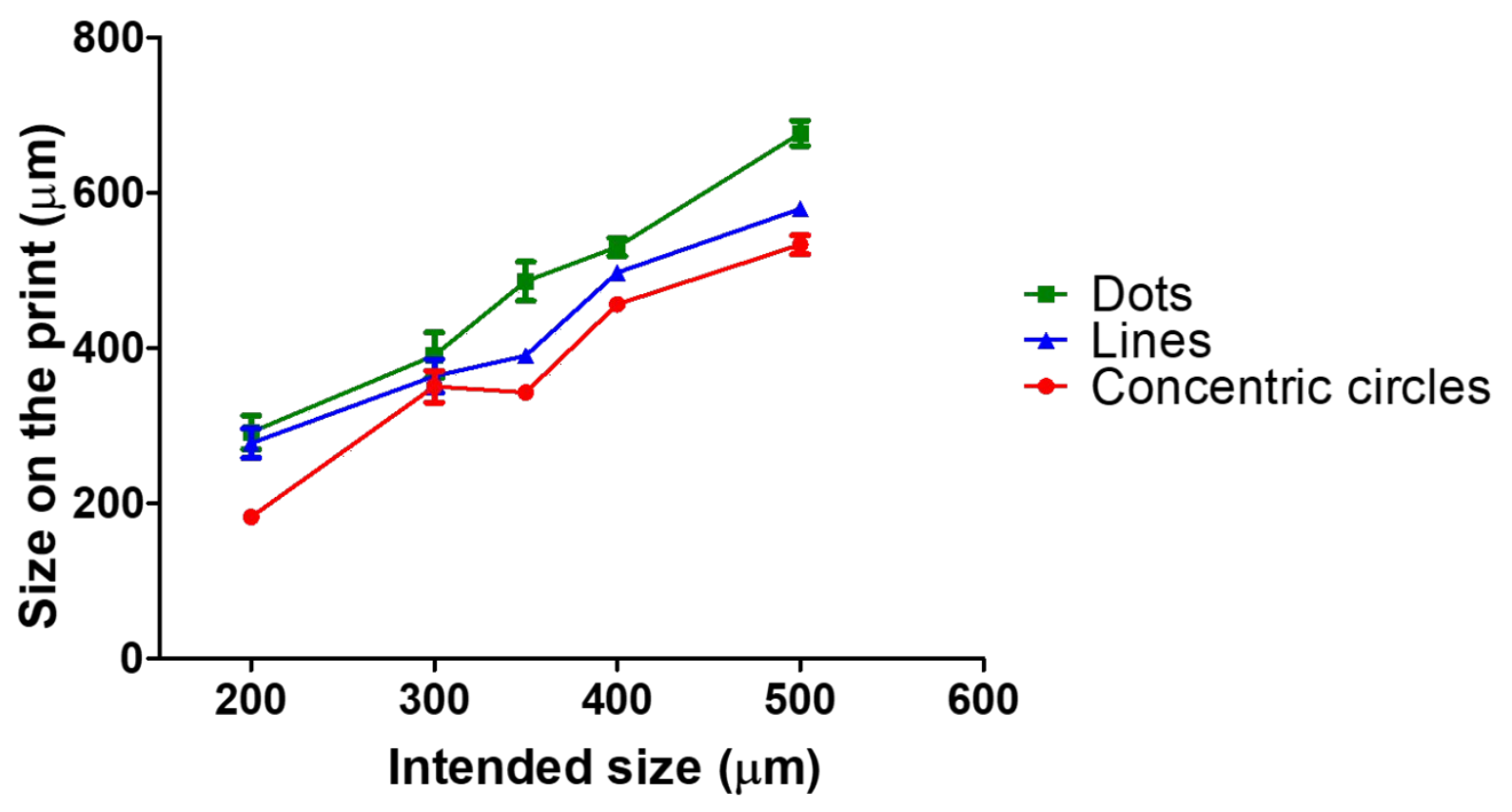


Supplementary Figure 3: Sizes of lines, dots and concentric circles printed on the transparent sheet, showing an increase in size of the prints, which is translated into the later parts of screen assembly (photomasking) and thereby effecting the sizes of the hydrogel prints

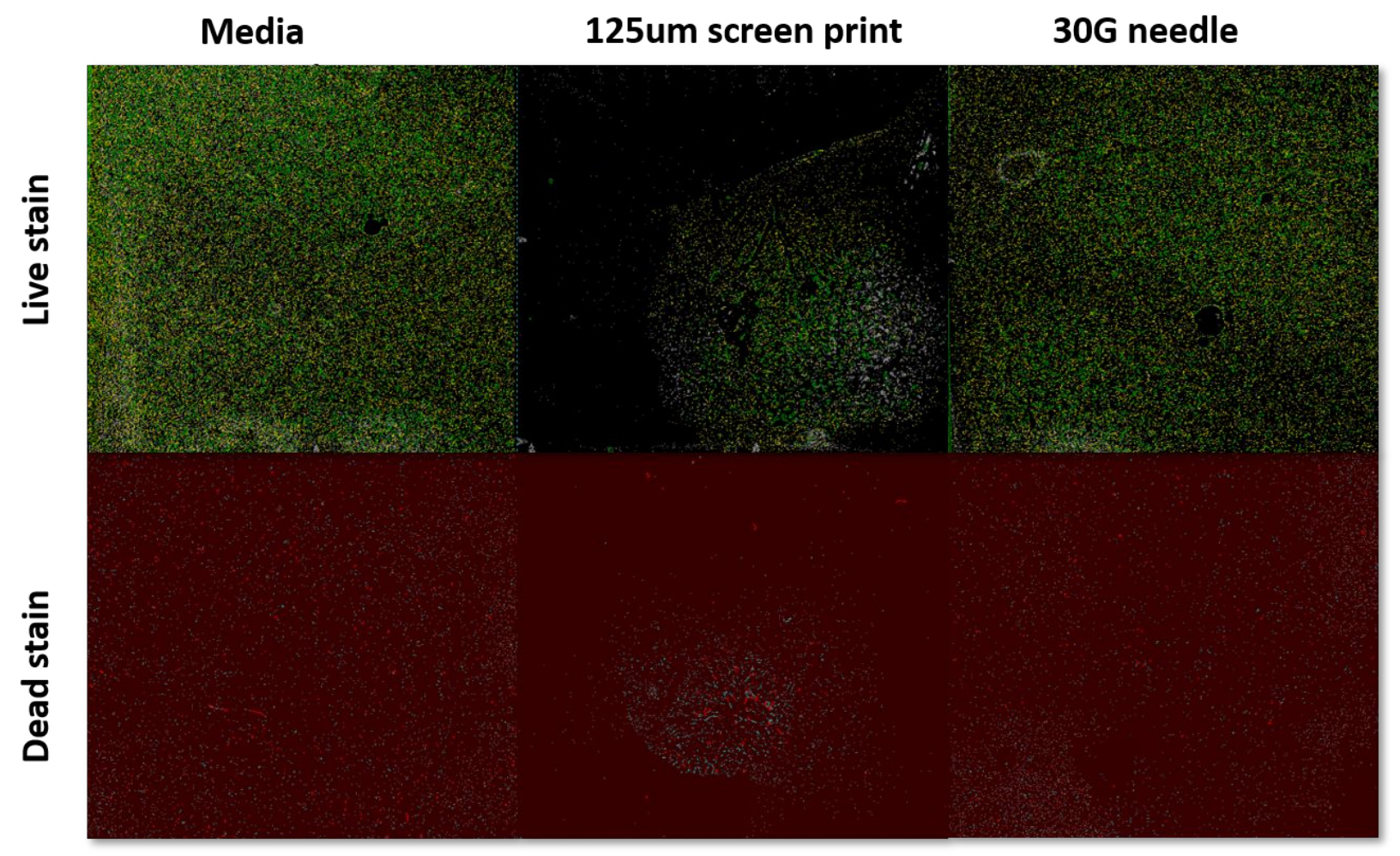

Supplementary Figure-4: Virtual images of Live/Dead scans of the Caco-2 cells printed using the screen printing techniques with a (125 $\mu \mathrm{m}$ mesh size of screen along with a control and 30G needle.

\section{AUTHOR INFORMATION \\ * Corresponding Author}

Erin Lavik

Email: elavik@umbc.edu 